\title{
A quantum autoencoder: using machine learning to compress qutrits
}

\author{
Alex Pepper, Nora Tischler, Geoff J. Pryde \\ Centre for Quantum Dynamics, Griffith University, Brisbane, QLD 4111, Australia \\ alex.pepper@griffithuni.edu.au
}

\begin{abstract}
The compression of quantum data will allow increased control over difficult-tomanage quantum resources. We experimentally realize a quantum autoencoder, which learns to compress quantum data with a classical machine learning routine.
\end{abstract}

(C) 2020 The Authors

Introduction: Quantum technology is a rapidly expanding area of interest, with Google and IBM both releasing prototype quantum devices. Whilst these types of devices are expected to provide an advantage over their classical counterparts, they depend on quantum resources which are difficult to produce, control, and effectively preserve over long distances. Thus, protocols that can reduce the requirements of these resources are highly valuable.

Classical and quantum technologies are not entirely dissimilar. Frequently, classical technology or architecture can be adapted to a similar quantum analog. The combination of machine learning and quantum information processing is a growing research area, which aims to either draw on classical machine learning techniques to aid quantum information tasks or utilize quantum information processing to speed up classical machine learning calculations. Of the former, one such device is the quantum autoencoder.

Classically, an autoencoder uses machine learning to encode information into a reduced number of dimensions, as illustrated in Fig. 1(a). Autoencoders form one of the core architectures in machine learning and offer a range of tools that primarily see applications in image processing. Multiple autoencoders that compress quantum data have been proposed, such as that in ref; [1]. Our quantum autoencoder is unique in that it looks to reduce the dimensions of single-photon qudits instead of, for example, mapping qubits to a lower number of qubits [2].

In this work, we apply a photonic quantum autoencoder to the task of compression. We consider families of qutrit states that are losslessly compressible to qubits. The device can be trained based on a few examples of the family of qutrit states, and can subsequently be tested with other qutrits from the family. Our autoencoder uses a classical machine learning algorithm, gradient descent, to optimize a unitary transformation for compressing the quantum states.

\section{Autoencoder:}

(a)

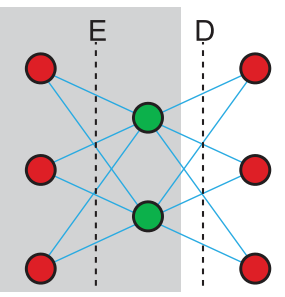

(b)

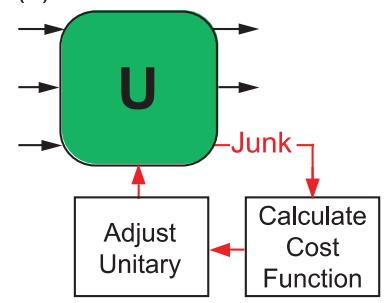

Fig. 1. The concept of an autoencoder [3]. (a) Through an encoding process (E), autoencoders represent data in a lower-dimensional space; if the compression is lossless, the original inputs can be perfectly recovered through a decoding process (D). (b) The scheme of our qudit-based autoencoder, equivalent to the grey shaded section in (a), for the case of compression of qutrits to qubits. A unitary transformation (U) characterized by a set of parameters, makes adjustments to the three input paths. For each iteration of the device, we calculate a cost function, which is the averaged probability of finding information in the junk output. This is then used to adjust the unitary. Lossless compression is achieved when the junk mode is unoccupied (adapted from [3]). 
We characterized our device's performance by looking at its compression rate, how that rate is affected by the number of training states, and its performance when subject to non-ideal channels. To test the compression rate we used two fixed, randomly selected training states to perform the optimization routine. An example of the cost function's value as the number of evaluations increases can be seen in Fig. 2.

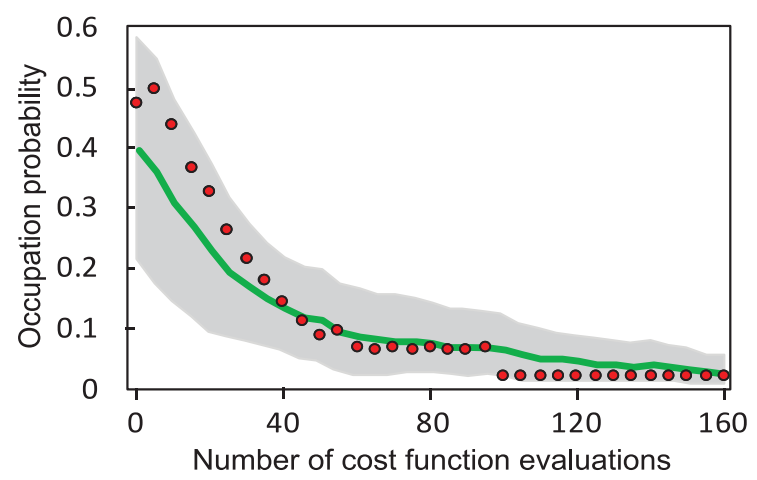

Fig. 2. Training starting from different initializations of the unitary. Here the compression over 20 training runs can be seen. Each of these runs started with a randomly generated unitary. The green line shows the average compression, the grey shaded area indicates how the compression varies, and the red dots illustrate a sample run (adapted from [3]).

We consistently observe near-lossless compression, that is, our autoencoder can effectively compress qutrits by exploiting the underlying structure of the dataset. We find that quantum autoencoders have an advantage over other methods of quantum data compression as they do not require specific assumptions about the quantum state. They can learn fixed structures based on a training dataset, making them a highly versatile tool.

\section{References:}

[1] J. Romero, J. P. Olson, and A. Aspuru-Guzik, "Quantum autoencoders for efficient compression of quantum data" in Quantum Science and Technology 2, 045001 (2017).

[2] Y. Ding, L. Lamata, M. Sanz, X. Chen, and E. Solano, "Experimental Implementation of a Quantum Autoencoder via Quantum Adders" in arXiv:1807.10643

[3] A. Pepper, N. Tischler, and G J. Pryde, "Experimental Realization of a Quantum Autoencoder: The Compression of Qutrits via Machine Learning" in Physical Review Letters 122, 060501 (2019).' 